\title{
TLDR: Extreme Summarization of Scientific Documents
}

\author{
Isabel Cachola $^{\dagger} \quad$ Kyle Lo $^{\dagger} \quad$ Arman Cohan $^{\dagger} \quad$ Daniel S. Weld $^{\dagger \dagger}$ \\ ${ }^{\dagger}$ Allen Institute for AI \\ ${ }^{\ddagger}$ Paul G. Allen School of Computer Science \& Engineering, University of Washington \\ $\{$ isabelc,kylel, armanc, danw\}@allenai.org
}

\begin{abstract}
We introduce TLDR generation, a new form of extreme summarization, for scientific papers. TLDR generation involves high source compression and requires expert background knowledge and understanding of complex domain-specific language. To facilitate study on this task, we introduce SCITLDR, a new multi-target dataset of $5.4 \mathrm{~K}$ TLDRs over $3.2 \mathrm{~K}$ papers. SCITLDR contains both author-written and expert-derived TLDRs, where the latter are collected using a novel annotation protocol that produces high-quality summaries while minimizing annotation burden. We propose CATTS, a simple yet effective learning strategy for generating TLDRs that exploits titles as an auxiliary training signal. CATTS improves upon strong baselines under both automated metrics and human evaluations. Data and code are publicly available at https://github.com/allenai/scitldr.
\end{abstract}

\section{Introduction}

We introduce TLDR ${ }^{1}$ generation for scientific papers. An alternative to abstracts, TLDRs focus on the key aspects of the paper, such as its main contributions, eschewing nonessential background or methodological details. Given the increasing pace of publication (Van Noorden, 2014) and resulting difficulty in keeping up with the literature, TLDRs can enable readers to quickly discern a paper's key points and decide whether it's worth reading. The goal of existing work in summarization of scientific documents is to generate abstracts or provide complimentary summaries to abstracts. (Collins et al., 2017; Cohan et al., 2018; Chandrasekaran et al., 2019; Yasunaga et al., 2019). In contrast, TLDR

\footnotetext{
${ }^{1}$ TLDR is an acronym that stands for "too long; didn't read," which is often used in online informal discussion (e.g., Twitter or Reddit) about scientific papers. For visual clarity, we omit the semi-colon.
}

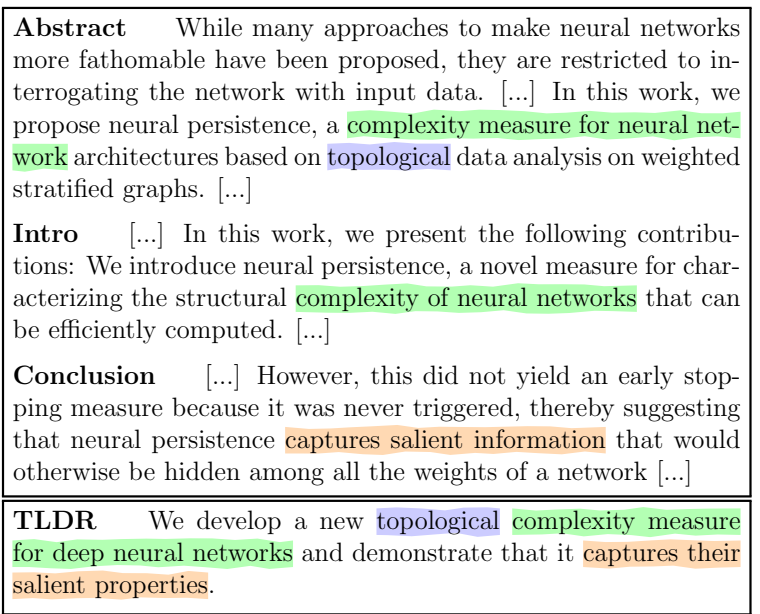

Figure 1: An example TLDR of a scientific paper. A TLDR is typically composed of salient information (indicated by colored spans) found in the abstract, intro, and conclusion sections of a paper.

generation seeks to produce an extreme (single sentence) summary (Narayan et al., 2018) given the entire paper. Further, TLDR generation is a challenging natural language generation task. Writing a TLDR of a scientific paper requires expert background knowledge and understanding of complex domain-specific language to identify the salient aspects of the paper, while maintaining faithfulness to the source and correctness of the written summary. An example TLDR is provided in Figure 1.

To facilitate the study of TLDR generation, we introduce SCITLDR, a new dataset of 5,411 TLDRs of computer science papers. SCITLDR is built from a combination of TLDRs written by authors of submissions on OpenReview ${ }^{2}$ and TLDRs derived by a novel annotation protocol that asks domain experts to rewrite peer review comments for that submission. Having multiple gold summaries per paper is especially important for evaluation when there is

\footnotetext{
${ }^{2}$ https://openreview.net/
} 
variability in human-written gold summaries (Zechner, 1996; Harman and Over, 2004).

In addition to establishing strong extractive and abstractive summarization baselines using Transformer-based (Vaswani et al., 2017) models, we present CATTS (Controlled Abstraction for

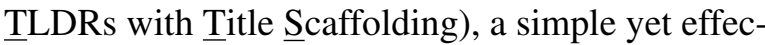
tive learning strategy for TLDR generation. CATTS incorporates ideas from scaffold tasks for multitask learning (Swayamdipta et al., 2018a; Cohan et al., 2019) and control codes in conditional language generation (Keskar et al., 2019) to address the problem of data scarcity in the highly-specialized scientific domain. In particular, CATTS exploits titles as an auxiliary, naturally-occurring training signal by training the model to generate both titles and TLDRs indicated by control codes. We show that CATTS applied to BART (Lewis et al., 2020), a state-of-the-art summarization model, results in performance improvement in both automated metrics and human evaluation.

Our contributions are summarized below:

1. We introduce TLDR generation, a new form of extreme summarization, for scientific papers. With extensive analysis of properties of TLDRs, we provide insight into the types of information and amount of variability in human-written TLDRs.

2. We release SCITLDR, a new multi-target dataset of 5,411 TLDRs over 3,229 scientific papers. SCITLDR contains both author-written and expertderived TLDRs, where the latter are collected using a novel annotation protocol that produces highquality summaries while avoiding the burden of reading the full paper.

3. We establish strong baselines on SCITLDR and improve them with CATTS, a simple yet effective learning strategy for generating TLDRs that uses titles as an auxiliary training signal.

4. We perform extensive analysis and human evaluation of system-generated TLDRs, focusing on informativeness and factual correctness.

\section{Dataset construction}

Overview We introduce SCITLDR, a new multitarget dataset of 5,411 TLDRs over 3,229 scientific papers in the computer science domain. ${ }^{3}$ The training set contains 1,992 papers, each with a single gold TLDR. The dev and test sets contain 619 and 618 papers each, with 1,452 and 1,967 TLDRs, respectively. This is unlike the majority of existing

\footnotetext{
${ }^{3}$ See Appendix Table 9 for full venue breakdown.
}

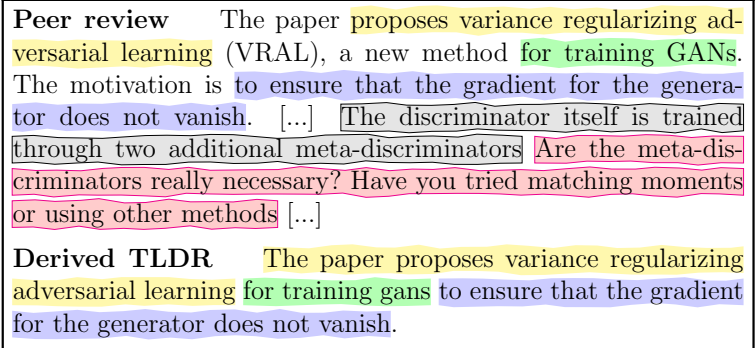

Figure 2: Example of a reviewer comment rewritten as a TLDR (best viewed in color). A peer review comment often begins with a summary of the paper which annotators use to compose a TLDR. Annotators are trained to preserve the original reviewer's wording when possible (indicated by colored spans), and to avoid using any excess details or criticism.

summarization datasets that assume only one gold summary for a given document.

As evidenced by earlier work in summarization evaluation (Cohan and Goharian, 2016), variability in human-written summaries (Zechner, 1996; Harman and Over, 2004) can negatively impact the reliability of automated summarization metrics like Rouge (Lin, 2004). ${ }^{4}$ Considering only one gold TLDR for each paper as a basis of automated evaluation might result in inaccurate system quality assessment because content that might appear in a TLDR can have large variability. In addition, having multiple gold summaries for each document enables performing more in-depth analysis and thorough evaluation (Nenkova and Passonneau, 2004).

To address this, SCITLDR contains TLDRs written from the perspective of the author ("TLDRAuth") and TLDRs written from the perspective of the peer reviewer("TLDR-PR"). We describe these two types of TLDRs in the following paragraphs.

Collecting TLDR-Auth pairs Scholar-written TLDRs of scientific papers are available on various online platforms. On OpenReview.org, a publicly available scientific reviewing platform, authors submit TLDRs of their papers that summarize the main content for both reviewers and other interested scholars. Scholars also share TLDRs social media platforms, such as Twitter and Reddit.

We use the OpenReview API $^{5}$ to collect pairs of papers and author-written TLDRs, along with the

\footnotetext{
${ }^{4}$ While Rouge is capable of handling multiple targets for a given document, most summarization datasets are single target. See Table 1.

${ }^{5}$ https://github.com/openreview/openreview-py
} 


\begin{tabular}{|c|c|c|c|c|c|c|}
\hline Dataset & $\begin{array}{l}\text { Number of } \\
\text { documents } i\end{array}$ & $\begin{array}{l}\text { Avg. words } \\
\text { in document }\end{array}$ & $\begin{array}{l}\text { Avg. words } \\
\text { in summary }\end{array}$ & $\begin{array}{r}\text { Compression } \\
\text { ratio }\end{array}$ & $\begin{array}{r}\% \text { novel } \\
\text { words }\end{array}$ & Multi-target \\
\hline \multicolumn{7}{|l|}{ Non-scientific documents } \\
\hline DUC (Over, 2003) & 624 & 441 & 11 & 40.1 & 30.0 & yes \\
\hline NYTimes (Sandhaus, 2008) & $655 \mathrm{~K}$ & 549 & 40 & 13.7 & 20.1 & no \\
\hline DailyMail (Hermann et al., 2015) & $220 \mathrm{~K}$ & 653 & 55 & 11.9 & 17.0 & no \\
\hline CNN (Hermann et al., 2015) & $93 \mathrm{~K}$ & 760 & 46 & 16.5 & 16.8 & no \\
\hline XSUM (Narayan et al., 2018) & $226 \mathrm{~K}$ & 431 & 23 & 18.7 & 35.8 & no \\
\hline Newsroom (Grusky et al.) & $1.32 \mathrm{M}$ & 659 & 27 & 24.4 & 26.0 & no \\
\hline BigPatent (Sharma et al., 2019) & $1.34 \mathrm{M}$ & $3.6 \mathrm{~K}$ & 117 & 30.5 & 13.6 & no \\
\hline \multicolumn{7}{|l|}{ Scientific documents } \\
\hline CLPubSum (Collins et al., 2017) & $10.3 \mathrm{~K}$ & $8.2 \mathrm{~K}$ & 226 & 36.5 & 7.7 & no \\
\hline PubMed (Cohan et al., 2018) & $133 \mathrm{~K}$ & $3 \mathrm{~K}$ & 203 & 14.9 & 10.5 & no \\
\hline ArXiv (Cohan et al., 2018) & $215 \mathrm{~K}$ & $4.9 \mathrm{~K}$ & 220 & 22.5 & 8.3 & no \\
\hline SciSummNet $^{\dagger}$ (Yasunaga et al., 2019) & $1.0 \mathrm{~K}$ & $4.7 \mathrm{~K}$ & 150 & 31.2 & 7.4 & no \\
\hline TalkSumm $^{\ddagger}$ (Lev et al., 2019) & $1.7 \mathrm{~K}$ & $4.8 \mathrm{~K}$ & 965 & 5.0 & 16.5 & no \\
\hline SCITLDR (ours) & $3.2 \mathrm{~K}$ & $5 \mathrm{~K}$ & 21 & 238.1 & 15.2 & yes \\
\hline
\end{tabular}

Table 1: Comparison of SCITLDR to existing summarization datasets. (i) SCITLDR provides multiple summary targets unlike other recent summarization datasets. (ii) SCITLDR requires both extreme compression and abstraction, as evidenced by the compression ratio and novelty (\% of summary words not in the source document), especially when compared with other scientific summarization datasets.

${ }^{\dagger}$ SciScummNet data was later included in the CL-SciSumm shared task and dataset (Jaidka et al., 2018; Chandrasekaran et al., 2019), which has an additional 40 manually annotated documents and its statistics are similar to SciSummNet.

${ }^{\ddagger}$ Unlike the other summarization datasets presented here, TalkSumm is an automatically-constructed dataset for training; the TalkSumm-supervised model in Lev et al. (2019) was evaluated using CL-SciSumm (Jaidka et al., 2018).

full-text $\mathrm{PDFs}^{6}$ of those papers. We use the S2ORC pipeline (Lo et al., 2020) to convert PDFs to structured, machine-readable full text. We then split the papers randomly into the previously-mentioned train, dev, and test sets; each paper at this point has an associated author-written gold TLDR.

Rewriting peer reviews into TLDR-PR pairs Scaling up data collection in a specialized scientific domain is costly and challenging. To sidestep this problem, we use a novel annotation protocol that exploits natural summaries in peer review comments. Assuming the typical peer reviewer has carefully scrutinized the source paper and provided a faithful summary in their comment (often in the first paragraph), domain experts can rewrite these comments into TLDRs.

For this task, we recruit 28 undergraduate computer science students from the University of Washington with self-reported experience in reading scientific papers. Each recruited student received one hour of one-on-one writing training and then was asked to work independently. Annotators were only

\footnotetext{
${ }^{6} \mathrm{~A}$ small fraction of those papers $(<5 \%)$ did not have an available PDF file, so we could not parse their full body text. This are still included the dataset as it is possible to generate a TLDR from an abstract alone.
}

shown the first 128 words of a sampled ${ }^{7}$ peer review comment. They were instructed to keep their TLDRs between 15-25 words (similar to the length of an author written TLDR) and to skip reviews that do not contain a summary or if they did not understand the content. They were also instructed to use the original language in the review, when possible. We manually assessed every written summary, discarding TLDRs that did not adhere to the guidelines, and allowed 20/28 students who performed well to continue work beyond the first hour. Students were compensated at the local median hourly wage of $\$ 20$ USD per hour. Refer to Appendix $\S \mathrm{F}$ for full annotation instructions. Figure 2 contains an example of a peer review and its corresponding TLDR-PR. We discuss differences between TLDRPR and TLDR-Auth throughout Section 3.

\section{Dataset analysis}

\subsection{Compression and abstractiveness}

Table 1 compares SCITLDR with other summarization datasets in both scientific and non-scientific domains. We observe that SCITLDR has short summaries, like XSUM and NewsRoom, with long

\footnotetext{
${ }^{7}$ Multiple peer review comments can be available for each paper on OpenReview. We focused on ensuring that each paper in dev and test had at least one TLDR-PR.
} 
source documents, like BigPatent and the other scientific-domain datasets. This results in a much higher compression ratio compared with existing datasets. Summarization in higher compression settings is challenging as it requires capturing more precisely the salient aspects of the document (Grusky et al.).

Following Narayan et al. (2018); Grusky et al., we measure abstractiveness (or novelty) by percentage of words in the summary that do not appear in the source document. We observe that SCITLDR is more abstractive compared with other scientific domain datasets but less abstractive compared with non-scientific domain datasets. We also observe that SCITLDR is smaller in comparison to automatically collected datasets, such as XSUM and ArXiv, but is larger in comparison to other manually collected datasets, such as SciSummNet.

\subsection{Information content}

We analyze the information content of TLDRs using an approach motivated by the nugget-based summarization evaluation framework of Nenkova and Passonneau (2004). In a similar manner, we asked two computer science researchers to read through a collection of TLDRs to both define a comprehensive set of categories of types of information present in TLDRs, which we refer to as nuggets. ${ }^{8}$ We also label each TLDR with all represented nuggets. Table 2 presents this categorization, along with example phrases and nugget occurrence frequencies of SCITLDR. For simplicity, we use the category codes defined in the table (with brackets) to reference specific categories.

Most TLDRs contain between two to four nuggets (never all six), and will provide some indication of their subject area (A) and the paper's contributions $(\mathbf{C})$. In fact, they are the most frequently co-occurring nuggets, appearing in $63 \%$ of TLDRAuth and $71 \%$ of TLDR-PR. TLDR-Auth tend to include results or scientific/theoretical findings $(\mathbf{R})$ and often signal the value of their work $(\mathbf{V})$ by describing their contributions as novel or their results as strong or state-of-the-art. In contrast, TLDR$\mathrm{PR}$ focus more on articulating problems the paper addresses (P). Interestingly, TLDR-PR place less emphasis on $\mathbf{R}$ and $\mathbf{V}$ in favor of further methodological details in the paper $\mathbf{D}$. More details about nuggets in Appendix $\S$ A.

\footnotetext{
${ }^{8}$ While we adopt the term "nugget" for convenience, we recognize that that they traditionally correspond to factoids, while here they correspond to discourse roles Teufel (1999).
}

\begin{tabular}{|c|c|c|}
\hline Category & Example phrase & $\begin{array}{r}\text { \% of TLDRs } \\
\text { AUTH / PR }\end{array}$ \\
\hline $\begin{array}{l}\text { [A]rea, field } \\
\text { or topic of study }\end{array}$ & $\begin{array}{l}\text { reinforcement learning, } \\
\text { dependency parsing }\end{array}$ & $85.6 / 90.8$ \\
\hline $\begin{array}{l}{[\mathbf{P}] \text { roblem or }} \\
\text { motivation }\end{array}$ & $\begin{array}{l}\text { mode collapse, } \\
\text { catastrophic forgetting }\end{array}$ & $29.0 / 32.9$ \\
\hline $\begin{array}{l}\text { Mode of } \\
\text { [C]ontribution }\end{array}$ & $\begin{array}{l}\text { method, dataset, } \\
\text { proof, theorem }\end{array}$ & $68.4 / 76.3$ \\
\hline $\begin{array}{l}\text { [D]etails or } \\
\text { description }\end{array}$ & $\begin{array}{l}\text { graph convolution } \\
\text { operations with dynam- } \\
\text { ically computed graphs }\end{array}$ & $43.4 / 57.9$ \\
\hline $\begin{array}{l}{[\mathbf{R}] \text { esults or }} \\
\text { findings }\end{array}$ & $\begin{array}{l}\text { improved performance } \\
\text { on ImageNet, } \\
\text { simple defenses work on } \\
\text { MNIST but not CIFAR }\end{array}$ & $29.0 / 17.1$ \\
\hline $\begin{array}{l}\text { [V]alue or } \\
\text { significance }\end{array}$ & $\begin{array}{l}\text { novel, state-of-the-art, } \\
\text { simple yet effective, } \\
\text { easily applicable }\end{array}$ & $23.7 / 7.9$ \\
\hline
\end{tabular}

Table 2: Example categories (or nuggets) of information a TLDR might contain. Proportion of TLDRs containing each nugget estimated on 76 randomly sampled gold papers (each with its TLDR-Auth and a sampled TLDR-PR). Percentages do not sum to one because each TLDR can contain multiple nuggets.

\subsection{Variability in TLDRs}

To explore variability in our human-written summaries, we examine differences between TLDRs written by authors (TLDR-Auth) and TLDRs derived from the perspective of a peer reviewer (TLDR-PR).

Lexical variation First, we note that TLDR-Auth are on average 18.9 words long, while TLDR-PR are slightly longer on average at 22.9 words. Despite similarities in length, the 1-, 2-, and 3-gram mean Jaccard indices between TLDR-Auth and TLDR-PR are $15.0 \%, 2.5 \%$, and $0.7 \%$, respectively, indicating extremely little lexical overlap between the two sources of TLDRs. We can also observe through qualitative examples in Figure 3 how TLDR-Auth and TLDR-PR can differ greatly, even when they contain the same information content.

Abstractiveness TLDR-PR is more abstractive with a novelty score of $20.2 \%$ compared with TLDRAuth with a novelty score of $9.6 \%$, where novelty is computed as the percentage of words in the TLDR not in the source paper. This is not unexpected because TLDR-PR are derived from peer review comments which themselves have already gone through one stage of abstraction. 


\begin{tabular}{l}
\hline TLDR-Auth The authors propose a framework to learn a \\
good policy through imitation learning from a noisy demonstra- \\
tion set via meta-training a demonstration suitability assessor. \\
TLDR-PR Contributes a maml based algorithm for imita- \\
tion learning which automatically determines if provided demon- \\
strations are "suitable". \\
\hline \hline TLDR-Auth The authors evaluate the effectiveness of hav- \\
ing auxiliary discriminative tasks performed on top of statistics \\
of the posterior distribution learned by variational autoencoders \\
to enforce speaker dependency. \\
TLDR-PR Propose an autoencoder model to learn a rep- \\
resentation for speaker verification using short-duration analysis \\
windows.
\end{tabular}

Figure 3: Two example TLDR-Auth and TLDR-PR pairs with colored spans corresponding to nuggets in Table 3 - A, P, C, D. On top, we see TLDRs can have substantial lexical variation despite covering similar information content. On bottom, we naturally see even more variation when the information content differs.

\section{Catts}

We introduce CATTS (Controlled Abstraction for TLDRs with Title $\underline{S}$ caffolding), a simple yet effective method for learning to generate TLDRs. Our approach addresses two main challenges: (1) the limited size of the training data and (2) the need for domain knowledge in order to write high-quality gold TLDRs. To address these challenges, we propose using titles of scientific papers as additional generation targets. As titles often contain key information about a paper, we hypothesize that training a model to generate titles will allow it to learn how to locate salient information in the paper that will be also useful for generating TLDRs. In addition, all papers have a title, and thus we have an abundant supply of paper-title pairs for training.

Incorporating auxiliary scaffold tasks via multitask learning has been studied before for improving span-labeling and text classification (Swayamdipta et al., 2018b; Cohan et al., 2019). Similar to multitask learning, training on heterogenous data annotated with control codes has been shown to improve controlled generation in autoregressive language models (Keskar et al., 2019; ElSahar et al., 2020; Sudhakar et al., 2019; Li et al., 2020). In fact, it has been shown effective for generating biomedical abstracts (Sybrandt and Safro, 2020). We demonstrate that control codes can be used to effectively incorporate scaffold tasks (e.g. title generation) for denoising autoencoders like BART (Lewis et al., 2020).

In order to use title generation as a scaffold task for TLDR generation, we propose shuffling

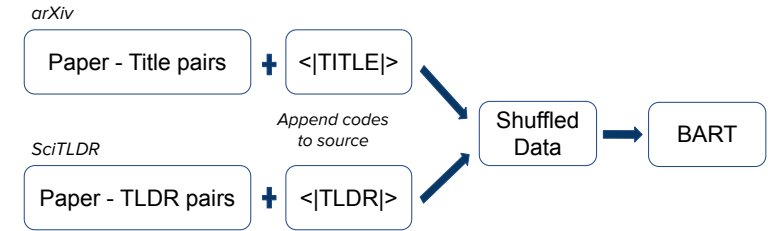

Figure 4: Training regimen for CATTS.

SCITLDR with a title generation dataset, then appending each source with control codes $\langle|T L D R|\rangle$ and $\langle|\operatorname{TITLE}|\rangle$, respectively. This allows the parameters of the model to learn to generate both TLDRs and titles. This process is visualized in Frigure 4. At generation time, the appropriate control code is appended to the source. Additionally, upsampling particular tasks can be viewed as applying task-specific weights, similar to weighting losses in multitask learning setups.

\section{Experiments}

\subsection{Baselines}

We establish baselines for TLDR generation on SCITLDR using state-of-the-art extractive and abstractive summarization models.

Extractive methods We consider both unsupervised and supervised extractive methods. For our unsupervised baseline, we use PACSUM (Zheng and Lapata, 2019), an extension of TextRank (Mihalcea and Tarau, 2004) that uses BERT (Devlin et al., 2019) as a sentence encoder. For our supervised baselines, we use BERTSUMEXT (Liu and Lapata, 2019), which uses BERT as a sentence encoder augmented with inter-sentence Transformer layers to capture interactions, and MatchSum (Zhong et al., 2020), which uses a BERT Siamese network to score whole summaries.

\footnotetext{
Abstractive methods Since TLDRs often contain information spread across multiple sentences, we expect abstractive summarization methods to produce strong results for this task. We focus on BART (Lewis et al., 2020), a Transformer-based denoising autoencoder for pretraining sequenceto-sequence models. We use BART-large, which achieves state-of-the-art results in summarization on XSUM. We additionally use BART-large finetuned on XSUM, hypothesizing that the task of extreme summarization of news articles might transfer to TLDR generation on SCITLDR.
} 
Oracle We define a sentence-level extractive oracle: Given a paper and its multiple gold TLDRs, it selects the single sentence in the document with the highest Rouge overlap for each gold TLDR. Then it returns the single sentence that yields the maximum Rouge across all gold TLDRs. This sets an upperbound on the performance of the sentence-level extractive methods under our multi-target evaluation (Section 5.4). Our full text oracle achieves 54.5 Rouge-1, 30.6 Rouge-2, and 45.0 Rouge-L on the test set.

\subsection{Input space}

The input space is the context provided to the model when generating TLDRs.

Abstract-only Since the vast majority of scientific papers do not have open-access full text (Lo et al., 2020), it is worth considering the setting in which we generate TLDRs for papers given only their abstracts as input. The average length of an abstract is 159 words and resulting compression ratio is 7.6.

AIC Previous studies have found that the most salient information in a paper for writing a summary is often found in the abstract, introduction, and conclusion (AIC) sections (Sharma et al., 2019). An important consequence of this is the ability to substantially reduce computational costs 9 (Schwartz et al., 2019) by supplying only these sections as context. The average combined length of these contexts is 993 words and resulting compression ratio is 47.3 , which is still higher than other datasets surveyed in Table 1.

Comparing oracle results in Table 3, we see that increasing the input space from abstract-only to AIC improves Rouge- 1 by +4.7 . Yet, this is only 2.1 Rouge-1 lower than the full text oracle performance, despite requiring five times more text.

\subsection{Training and implementation details}

All experiments use Titan V or V100 GPUs. We experiment on abstract-only and AIC input spaces. Best hyperparameters for the models are selected based on dev set Rouge-1. Supervised models like BERTSUMEXT and BART are trained on SCITLDR and the best model checkpoint chosen using dev set loss. See Appendix $\S D$ for additional parameter tuning details of all models.

\footnotetext{
${ }^{9}$ Especially for methods that rely on $O\left(n^{2}\right)$ inter-sentence comparisons or wrappers around Transformer-based methods to long contexts.
}

Extractive Methods For PACSUM, BERTSUMEXT and MatchSum we use original code released by the authors. The first two use BERT-base and the last one uses RoBERTa-base (Liu et al., 2019). For MatchSum in AIC input space, following the authors, we use BERTSUMEXT to first extract 7 highly scoring sentences as the input to MatchSum. ${ }^{10}$ Sentence segmentation is performed using ScispaCy (Neumann et al., 2019), and models select a single sentence as their predictions. We use the default hyperparameters for PACSUM.

Abstractive Methods We experiment with BART-large and BART-large finetuned on XSUM, using the Fairseq (Ott et al., 2019) implementation and the released XSUM weights. We apply the CATTS training method to these two models, using an additional $20 \mathrm{~K}$ paper-title pairs from arXiv for title generation. ${ }^{11}$ We up-sample TLDR instances to match the size of the title scaffold data. ${ }^{12}$ For simplicity, we refer to these as BART, BART $\mathrm{XSUM}_{\text {, }}$ CATTS and CATTSXSUM, respectively. For all models, we use a learning rate of $3 e-5$, update frequency of 1 , and max tokens per batch of $1024^{13}$ chosen through manual tuning. We tune decoder for all models via grid search over five length penalties between 0.2 and 1.0 and 7 beam sizes 2 to 8 .

\subsection{Evaluation}

Automated evaluation Following recent work on extreme summarization (Narayan et al., 2018; Lewis et al., 2020), we use Rouge-1, Rouge-2, and Rouge-L (Lin, 2004) as our automated metrics. As discussed in Section 2, we have multiple target summaries available per paper. To exploit this during evaluation, we calculate the Rouge score of the system-generated TLDR with respect to each of the gold TLDRs for the corresponding paper (including its TLDR-Auth and all of its TLDRS-PR) individually. We take the maximum Rouge score over these gold TLDRs as the final Rouge score for that paper. An alternative approach to aggregating scores would be to take the mean, but due to the

\footnotetext{
${ }^{10}$ In abstract-only setting, MatchSum takes the full context.

${ }^{11}$ Includes all papers on arXiv with at least one of the following tags CS.CL, CS.CV, CS.LG, CS.AI, CS.NE, and STAT.ML and have identified introduction and conclusion sections by S2ORC (Lo et al., 2020).

${ }^{12}$ While this up-sampling may indicate that CATTS is training on more TLDRs than BART, we allow BART training up to 20 epochs and it quickly overfits within a few epochs.

${ }^{13}$ Fairseq reports an "average batch size" of 36 , which is a consequence of adaptive batching of examples based on the update frequency and max tokens per batch.
} 


\begin{tabular}{|c|c|c|c|c|c|c|}
\hline \multirow[b]{2}{*}{ Method } & \multicolumn{3}{|c|}{ Abstract-only } & \multicolumn{3}{|c|}{ AIC } \\
\hline & $\mathrm{R} 1$ & $\mathrm{R} 2$ & RL & $\mathrm{R} 1$ & $\mathrm{R} 2$ & RL \\
\hline Oracle & 47.7 & 24.7 & 38.5 & 52.4 & 29.0 & 42.9 \\
\hline PACSUM (Zheng and Lapata, 2019) & 19.3 & 4.0 & 15.1 & 28.7 & 9.8 & 21.9 \\
\hline BERTSUMEXT (Liu and Lapata, 2019) & 38.5 & 16.6 & 30.5 & 36.2 & 14.7 & 28.5 \\
\hline MatchSum (Zhong et al., 2020) & 42.7 & 20.0 & 34.0 & 38.6 & 16.4 & 30.1 \\
\hline BART (Lewis et al., 2020) & 43.3 & 20.8 & 35.0 & 42.9 & 20.8 & 35.1 \\
\hline BART $_{\text {XSUM }}$ (Lewis et al., 2020) & 42.5 & 21.1 & 34.9 & 43.7 & 21.4 & 36.0 \\
\hline CATTS (Ours) & 43.8 & 20.9 & 35.5 & ${ }^{\dagger} 44.9$ & ${ }^{\dagger} 22.6$ & ${ }^{\dagger} 37.3$ \\
\hline CATTSXSUM (Ours) & ${ }^{\dagger} 44.3$ & 21.3 & 35.9 & 44.6 & 21.7 & 36.5 \\
\hline
\end{tabular}

Table 3: Test set max Rouge scores of extractive and abstractive baselines and CATTS. We use ${ }^{\dagger}$ to indicate CATTS variants that significantly $(p<0.05)$ outperform their corresponding BART baseline.

variability in TLDRs shown in Section 3.3, we argue the maximum operation is more appropriate - That is, matching any of the gold TLDRs is rewarded. ${ }^{14}$

Human evaluation While our multi-target setting allows us to mitigate some of the limitations of Rouge (Conroy et al., 2011; Cohan and Goharian, 2016), we acknowledge that relying only on automated metrics is insufficient for evaluating the quality of the models. In addition to automated metrics, we also have human experts in computer science assess system-generated TLDRs under two criteria - informativeness and correctness.

For informativeness, we perform the nuggetbased analysis for information content over systemgenerated TLDRs for the same 76 gold papers from Section 3.2. We use the presence (or lack) of different nuggets in predicted and gold TLDRs to quantify differences in information content. Specifically, we score each gold and system-generated TLDR by the number of unique nuggets divided by the number of tokens. This length normalization handles cases where systems returning the source document are trivially more informative. For each paper, we rank the predicted and gold TLDRs. Then, we compute overall metrics for each gold or system variant by aggregating their ranks across papers using mean reciprocal rank (MRR).

Evaluating correctness requires careful reading and understanding the source paper. To minimize this burden and have reliable evaluation, we ask the original authors of papers to assess the correctness of our system-generated TLDRs. We manually email (first or second) authors of arXiv papers and ask them to score each system-generated TLDR

\footnotetext{
${ }^{14}$ For completeness we provide mean Rouge scores in Appendix Table 10 to supplement our main max Rouge results in Table 3.
}

\begin{tabular}{lrrc}
\hline & MRR & $\begin{array}{l}\text { Avg. \# } \\
\text { nuggets }\end{array}$ & $\begin{array}{l}\text { Avg. \# } \\
\text { words }\end{array}$ \\
\hline TLDR-Auth (Gold) & 0.53 & 2.5 & 20.5 \\
TLDR-PR (Gold) & 0.60 & 2.4 & 18.7 \\
BART XSUM & 0.42 & 2.2 & 19.4 \\
CATTS XSUM & 0.54 & 2.6 & 20.8 \\
\hline
\end{tabular}

Table 4: Human evaluation on informativeness of gold and system-generated TLDRs. Higher MRR corresponds to variants that, on average, rank higher than others by length-normalized number of nuggets.

with 1 - false or misleading, 2 - partially accurate or 3 - mostly correct, regardless of comprehensiveness. We compare the mean correctness (across papers) for each system variant. We received responses from 29 unique authors with annotations covering 64 arXiv papers.

\section{Results}

\subsection{Quantitative results}

We present our main results in Table 3.

Extractive results We establish baseline results for extractive methods on our new dataset SCITLDR. We observe that MatchSum has the highest extractive performance, followed by BERTSUMEXT. We observe that increasing input space from abstract-only to AIC greatly improves PACSUM $^{15}$ performance but decreases performance of both BERTSUMEXT and MatchSum. We suspect that increasing the input space makes it more difficult for these models to learn optimal parameters including new position embeddings in low-resource training. Compared to the extractive oracle scores, we see there is plenty of room for improvement.

\footnotetext{
${ }^{15}$ PACSUM using the full text yields a Rouge- 1 of 12.7 , significantly worse than abstract-only.
} 


\begin{tabular}{lccccc}
\hline & \multicolumn{2}{c}{ Abstract-only } & & \multicolumn{2}{c}{ AIC } \\
\cline { 2 - 3 } \cline { 6 - 6 } Method & $\begin{array}{c}\text { \% novel } \\
\text { words }\end{array}$ & $\begin{array}{c}\text { Avg. \# } \\
\text { words }\end{array}$ & & $\begin{array}{c}\text { \% novel } \\
\text { words }\end{array}$ & $\begin{array}{c}\text { Avg. \# } \\
\text { words }\end{array}$ \\
\hline BART & $2.9 \%$ & 20.9 & & $1.3 \%$ & 20.4 \\
BART $_{\text {XSUM }}$ & $3.7 \%$ & 18.4 & & $1.1 \%$ & 18.9 \\
CATTS $^{\text {CATTS }}$ & $5.5 \%$ & 19.1 & & $5.3 \%$ & 18.4 \\
CASUM & $5.8 \%$ & 19.7 & & $4.5 \%$ & 19.7 \\
\hline
\end{tabular}

Table 5: Lexical features of system-generated TLDRs.

\begin{tabular}{lrrrrrr}
\hline Method & R1 & $\Delta$ & R2 & $\Delta$ & RL & $\Delta$ \\
\hline BART & 44.9 & +1.6 & 22.6 & +1.8 & 37.1 & +2.1 \\
BART $_{\text {XSUM }}$ & 44.8 & +1.1 & 21.8 & +0.4 & 36.4 & +0.4 \\
CATTS $_{\text {CATTS XSUM }}$ & 44.9 & +0.0 & 21.9 & -0.7 & 36.6 & -0.7 \\
\hline
\end{tabular}

Table 6: Oracle input space experiments. $\Delta$ are differences between oracle result and model's best performance (across abstract-only and AIC) from Table 3.

Abstractive results Abstractive methods are not limited to choosing exact sentences. For a given abstractive baseline BART or BART XSUM $_{\text {, our }}$ CATTS learning strategy results in improvements in both abstract-only and AIC settings. Comparing CATTS variants with their corresponding BART baselines, we observe that in the abstract-only setting, CATTS and CATTS XSUM achieve +0.5 and +1.8 Rouge- 1 , respectively. In the AIC setting, CATTS and CATTSXSUM achieve +2.0 and +0.9 Rouge-1, respectively. We use the two-sided paired t-test against a null hypothesis of no difference to assess these differences. To address the issue of multiple hypothesis testing over Rouge scores, we perform a Holm-Bonferroni (Holm, 1979) ${ }^{16}$ correction for determining significant $p$-values in Table 3 .

\subsection{Human evaluation}

We perform our human evaluation on BART $\mathrm{XSUM}_{\mathrm{X}}$ and CATTS XSUM using the AIC input space on 51 sampled papers. In this setting, we have both chosen the strongest baseline and controlled for XSUM pretraining. From Table 4, we see that CATTSXSUM is more informative than BART $\mathrm{XSUM}_{\text {and is com- }}$ parable to gold TLDR-Auth, though still less informative than TLDR-PR.

In addition to informativeness, we also evaluate content accuracy of generated tldrs as explained in Section 5.4. We report no difference in correctness

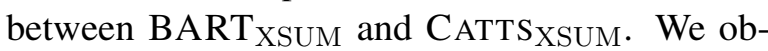
serve 42 ties, 10 cases where BART $\mathrm{XSUM}_{\mathrm{X}}$ is more correct, and 12 cases where CATTS XSUM is more

\footnotetext{
${ }^{16}$ Using the P.ADJUST library in R (R Core Team, 2018)
}

correct. Both models average a rating of 2.5 (scoring between partially accurate and mostly correct).

\subsection{Analysis}

How abstractive are the generations? From Table 5, we observe: (1) BART variants are less abstractive than CATTS variants. (2) Initial training on XSUM might influence models to be slightly less abstractive. (3) BART variants are more abstractive in the abstract-only setting than the longer AIC settings, while CATTS seems to have the same level of abstractiveness regardless of input space.

How long are the generations? From Table 5, we see the systems all generate TLDRs of similar length to the average length reported in Table 1.

How important is using the full text? To analyze whether one can improve abstractive model performance by improving the input space selection (compared to just using AIC), we define an oracle input space. That is, for each TLDR, we select sentences from the full text that maximize Rouge1 with the gold TLDRs-Auth ${ }^{17}$ and select the top sentences to match the length of AIC. Repeating the experiments in Section 5 with this input source, we observe some performance improvement across models (Table 6).

Qualitative example Table 7 contains system generations on the same paper (alongside the gold TLDRs). Curiously, despite both achieving the same Rouge-1, the generated TLDRs are quite different. BART $_{\text {XSUM }}$ focuses on the methodological contribution while CATTS XSUM focuses on a scientific finding. The "two hidden layer" detail by BART $_{\mathrm{XSUM}}$ is from the paper introduction and the "defining the appropriate sampling distributions" from CATTSXSUM is from the conclusion. ${ }^{18}$

\section{Related work}

Transformers for summarization Transformerbased models have achieved strong results in extractive and abstractive summarization. PACSUM (Zheng and Lapata, 2019) combines BERT sentence representation with unsupervised text ranking; MatchSum (Zhong et al., 2020) uses a Siamese BERT model to score the entire summary instead of a single extraction; and Liu and Lapata (2019)

\footnotetext{
${ }^{17}$ Only TLDRs-Auth is exists for all papers. TLDRs-PR are only in dev and test.

${ }^{18}$ See original paper:

https://openreview.net/pdf?id=SkGT6sRcFX
} 
TLDR-Auth We propose a method for the construction of arbitrarily deep infinite-width networks, based on which we derive a novel weight initialisation scheme for finite-width networks and demonstrate its competitive performance.

TLDR-PR Proposes a weight initialization approach to enable infinitely deep and infinite-width networks with experimental results on small datasets.

BART $_{\text {XSUM }}$ We propose a principled approach to weight initialisation that allows the construction of infinite-width networks with more than two hidden layers.

CATTS XSUM $_{\text {Xe study the initialisation requirements of }}$ infinite-width networks and show that the main challenge for constructing them is defining the appropriate sampling distributions for the weights.

Table 7: Examples of system generations. BART XSUM $_{\text {M }}$

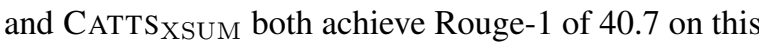
paper. Colored spans indicate text overlap.

show that BERT is effective for both extractive and abstractive summarization. Zhang et al. (2019); Bi et al. (2020) introduce new pretraining objectives that improve generation. Sequence-to-sequence models (Raffel et al., 2020; Lewis et al., 2020; Bao et al., 2020) have state-of-the-art performance on XSUM (Narayan et al., 2018), a dataset for extreme summarization dataset of news articles. SCITLDR is a new form of extreme summarization focused on scientific papers.

Scientific document summarization Most work in summarization of scientific papers have focused on longer summaries (i.e. 150-200 words). Existing datasets include CSPubSum for extractive summarization (Collins et al., 2017), ArXiv and PubMed for abstract generation (Cohan et al., 2018), and SciSummNet (Yasunaga et al., 2019) and CL-SciSumm (Jaidka et al., 2018; Chandrasekaran et al., 2019) datasets, which incorporate citation contexts into human-written summaries. TalkSumm (Lev et al., 2019) uses recordings of conference talks to create a distantly-supervised training set for the CL-SciSumm task.

Modeling approaches in scientific document summarization include models that exploit citation contexts (Qazvinian et al., 2013; Cohan and Goharian, 2015, 2017; Zerva et al., 2020), automated survey generation (Mohammad et al., 2009; Jha et al., 2015; Fabbri et al., 2018; Wang et al., 2018), and other techniques focusing on exploiting the unique properties of scientific documents such as long length and structure (Conroy and Davis, 2017; Nikolov et al., 2018; Cohan et al., 2018; Xiao and Carenini, 2019). Yet, such methods have not been studied in the setting of extreme summarization (i.e. short target summaries, high compression, high abstraction), and SCITLDR is the first dataset to facilitate such research.

\section{Conclusion}

We introduce TLDR generation for scientific papers, and release SCITLDR, a multi-target dataset of TLDR-paper pairs. We also present CATTS, a simple yet effective learning strategy for improving TLDR generation that exploits auxiliary training signal from paper titles. We show that our approach improves over strong modeling baselines.

Existing methods for scientific document summarization often make use of properties unique to those papers, like sections, citation contexts or scientific discourse roles. Future work can examine how best to incorporate these properties to improve TLDR generation models. Additionally, while our experiments are limited to abstract-only and AIC input spaces, we provide the full text of the source papers to support research into using longer input contexts. Furthermore, the multiple target summaries in SCITLDR reflect diverse perspectives and can be used to support summarization research into training and evaluation techniques previously unavailable with existing datasets. Finally, the idea of a TLDR can differ between academic disciplines, and we leave such exploration open for future work.

\section{Acknowledgments}

We thank the Semantic Scholar Research team and John Bohannon and Oleg Vasilyev from Primer for helpful feedback and discussions. This work was supported in part by NSF Convergence Accelerator award 1936940, NSF RAPID award 2040196, ONR grant N00014-18-1-2193, and the University of Washington WRF/Cable Professorship.

\section{References}

Hangbo Bao, Li Dong, Furu Wei, Wenhui Wang, Nan Yang, Xiulei Liu, Yu Wang, Songhao Piao, Jianfeng Gao, Ming Zhou, and Hsiao-Wuen Hon. 2020. Unilmv2: Pseudo-masked language models for unified language model pre-training. ArXiv, abs/2002.12804.

Bin Bi, Chenliang Li, Chen Wu, Ming Yan, and Wei Wang. 2020. Palm: Pre-training an autoencoding and autoregressive language model for contextconditioned generation. ArXiv, abs/2004.07159. 
Muthu Kumar Chandrasekaran, Michihiro Yasunaga, Dragomir Radev, Dayne Freitag, and Min-Yen Kan. 2019. Overview and results: Cl-scisumm shared task 2019. In Workshop on Bibliometric-enhanced Information Retrieval and NLP for Digital Libraries (BIRNDL)

Arman Cohan, Waleed Ammar, Madeleine van Zuylen, and Field Cady. 2019. Structural scaffolds for citation intent classification in scientific publications. In NAACL-HLT.

Arman Cohan, Franck Dernoncourt, Doo Soon Kim, Trung Bui, Seokhwan Kim, Walter Chang, and Nazli Goharian. 2018. A discourse-aware attention model for abstractive summarization of long documents. In NAACL-HLT.

Arman Cohan and Nazli Goharian. 2015. Scientific article summarization using citation-context and article's discourse structure. In EMNLP.

Arman Cohan and Nazli Goharian. 2016. Revisiting summarization evaluation for scientific articles. ArXiv, abs/1604.00400.

Arman Cohan and Nazli Goharian. 2017. Scientific document summarization via citation contextualization and scientific discourse. International Journal on Digital Libraries, 19:287-303.

Ed Collins, Isabelle Augenstein, and Sebastian Riedel. 2017. A supervised approach to extractive summarisation of scientific papers. CoNLL, abs/1706.03946.

John M. Conroy and Sashka Davis. 2017. Section mixture models for scientific document summarization. IJDL, 19:305-322.

John M Conroy, Judith D Schlesinger, and Dianne P O'Leary. 2011. Nouveau-rouge: A novelty metric for update summarization. Computational Linguistics, 37(1):1-8.

Jacob Devlin, Ming-Wei Chang, Kenton Lee, and Kristina Toutanova. 2019. Bert: Pre-training of deep bidirectional transformers for language understanding. ArXiv, abs/1810.04805.

Hady ElSahar, Maximin Coavoux, Matthias Gallé, and Jos Rozen. 2020. Self-supervised and controlled multi-document opinion summarization. ArXiv, abs/2004.14754.

Alexander Fabbri, Irene Li, Prawat Trairatvorakul, Yijiao He, Weitai Ting, Robert Tung, Caitlin Westerfield, and Dragomir Radev. 2018. TutorialBank: A manually-collected corpus for prerequisite chains, survey extraction and resource recommendation. In $A C L$.

Max Grusky, Mor Naaman, and Yoav Artzi. Newsroom: A dataset of 1.3 million summaries with diverse extractive strategies. In NAACL-HLT.
Donna Harman and Paul Over. 2004. The effects of human variation in DUC summarization evaluation. In Text Summarization Branches Out, pages 10-17, Barcelona, Spain. Association for Computational Linguistics.

Karl Moritz Hermann, Tomas Kocisky, Edward Grefenstette, Lasse Espeholt, Will Kay, Mustafa Suleyman, and Phil Blunsom. 2015. Teaching machines to read and comprehend. In Advances in neural information processing systems, pages 1693-1701.

Sture Holm. 1979. A simple sequentially rejective multiple test procedure. Scandinavian Journal of Statistics, 6(2):65-70.

Kokil Jaidka, Muthu Kumar Chandrasekaran, Sajal Rustagi, and Min-Yen Kan. 2018. Insights from clscisumm 2016: the faceted scientific document summarization shared task. IJDL, 19(2-3):163-171.

Rahul Jha, Reed Coke, and Dragomir R. Radev. 2015. Surveyor: A system for generating coherent survey articles for scientific topics. In $A A A I$.

Nitish Shirish Keskar, Bryan McCann, Lav R. Varshney, Caiming Xiong, and Richard Socher. 2019. CTRL: A Conditional Transformer Language Model for Controllable Generation. ArXiv, abs/1909.05858.

Guy Lev, Michal Shmueli-Scheuer, Jonathan Herzig, Achiya Jerbi, and David Konopnicki. 2019. Talksumm: A dataset and scalable annotation method for scientific paper summarization based on conference talks. In $A C L$.

Mike Lewis, Yinhan Liu, Naman Goyal, Marjan Ghazvininejad, Abdelrahman Mohamed, Omer Levy, Ves Stoyanov, and Luke Zettlemoyer. 2020. Bart: Denoising sequence-to-sequence pre-training for natural language generation, translation, and comprehension. ACL.

Kun Li, Chengbo Chen, Xiaojun Quan, Qing Ling, and Yan Song. 2020. Conditional augmentation for aspect term extraction via masked sequence-tosequence generation. ArXiv, abs/2004.14769.

Chin-Yew Lin. 2004. ROUGE: A package for automatic evaluation of summaries. In Text Summarization Branches Out, pages 74-81, Barcelona, Spain. Association for Computational Linguistics.

Yang Liu and Mirella Lapata. 2019. Text summarization with pretrained encoders. In EMNLP/IJCNLP.

Yinhan Liu, Myle Ott, Naman Goyal, Jingfei Du, Mandar Joshi, Danqi Chen, Omer Levy, Mike Lewis, Luke Zettlemoyer, and Veselin Stoyanov. 2019. Roberta: A robustly optimized bert pretraining approach. arXiv preprint arXiv:1907.11692.

Kyle Lo, Lucy Lu Wang, Mark Neumann, Rodney Kinney, and Daniel S. Weld. 2020. S2orc: The semantic scholar open research corpus. In Proceedings of $A C L$. 
Rada Mihalcea and Paul Tarau. 2004. Textrank: Bringing order into texts. In EMNLP.

Saif M. Mohammad, Bonnie J. Dorr, Melissa Egan, Ahmed Hassan Awadallah, Pradeep Muthukrishnan, Vahed Qazvinian, Dragomir R. Radev, and David M. Zajic. 2009. Using citations to generate surveys of scientific paradigms. In $H L T-N A A C L$.

Shashi Narayan, Shay B. Cohen, and Mirella Lapata 2018. Don't give me the details, just the summary! topic-aware convolutional neural networks for extreme summarization. pages 1797-1807.

Ani Nenkova and Rebecca J Passonneau. 2004. Evaluating content selection in summarization: The pyramid method. In NAACL.

Mark Neumann, Daniel King, Iz Beltagy, and Waleed Ammar. 2019. Scispacy: Fast and robust models for biomedical natural language processing.

Nikola I. Nikolov, Michael Pfeiffer, and Richard H. R Hahnloser. 2018. Data-driven summarization of scientific articles. ArXiv, abs/1804.08875.

Myle Ott, Sergey Edunov, Alexei Baevski, Angela Fan, Sam Gross, Nathan Ng, David Grangier, and Michael Auli. 2019. fairseq: a fast, extensible toolkit for sequence modeling. In NAACL-HLT, Demonstrations.

Paul Over. 2003. An introduction to duc 2003: Intrinsic evaluation of generic news text summarization systems. In Proceedings of Document Understanding Conference 2003.

Vahed Qazvinian, Dragomir R. Radev, Saif M. Mohammad, Bonnie J. Dorr, David M. Zajic, Michael Whidby, and Taesun Moon. 2013. Generating extractive summaries of scientific paradigms. J. Artif. Intell. Res., 46:165-201.

R Core Team. 2018. R: A Language and Environment for Statistical Computing. R Foundation for Statistical Computing, Vienna, Austria.

Colin Raffel, Noam Shazeer, Adam Roberts, Katherine Lee, Sharan Narang, Michael Matena, Yanqi Zhou, Wei Li, and Peter J. Liu. 2020. Exploring the limits of transfer learning with a unified text-to-text transformer. $J M L R, 21(140): 1-67$.

Evan Sandhaus. 2008. The new york times annotated corpus.(october 2008). ldc catalog no.: Ldc2008t19.

Roy Schwartz, Jesse Dodge, Noah A. Smith, and Oren Etzioni. 2019. Green ai.

Eva Sharma, Chen Li, and Lu Wang. 2019. Bigpatent: A large-scale dataset for abstractive and coherent summarization. In $A C L$.

Akhilesh Sudhakar, Bhargav Upadhyay, and Arjun Maheswaran. 2019. "transforming" delete, retrieve, generate approach for controlled text style transfer.
In Proceedings of the 2019 Conference on Empirical Methods in Natural Language Processing and the 9th International Joint Conference on Natural Language Processing (EMNLP-IJCNLP), pages 32693279, Hong Kong, China. Association for Computational Linguistics.

Swabha Swayamdipta, Sam Thomson, Kenton Lee, Luke Zettlemoyer, Chris Dyer, and Noah A. Smith. 2018a. Syntactic scaffolds for semantic structures. In $E M N L P$.

Swabha Swayamdipta, Sam Thomson, Kenton Lee, Luke Zettlemoyer, Chris Dyer, and Noah A. Smith. 2018b. Syntactic scaffolds for semantic structures. In EMNLP.

Justin Sybrandt and Ilya Safro. 2020. Cbag: Conditional biomedical abstract generation. ArXiv, $\mathrm{abs} / 2002.05637$.

S. Teufel. 1999. Argumentative zoning information extraction from scientific text.

Richard Van Noorden. 2014. Global scientific output doubles every nine years. Nature news blog.

Ashish Vaswani, Noam Shazeer, Niki Parmar, Jakob Uszkoreit, Llion Jones, Aidan N Gomez, Ł ukasz Kaiser, and Illia Polosukhin. 2017. Attention is all you need. In I. Guyon, U. V. Luxburg, S. Bengio, H. Wallach, R. Fergus, S. Vishwanathan, and R. Garnett, editors, NeurIPS.

Jie Wang, Chengzhi Zhang, Mengying Zhang, and Sanhong Deng. 2018. Citationas: A tool of automatic survey generation based on citation content. Journal of Data and Information Science, 3(2):20-37.

Wen Xiao and Giuseppe Carenini. 2019. Extractive summarization of long documents by combining global and local context. In EMNLP/IJCNLP.

Michihiro Yasunaga, Jungo Kasai, Rui Zhang, Alexander Richard Fabbri, Irene Li, Dan Friedman, and Dragomir R. Radev. 2019. Scisummnet: A large annotated corpus and content-impact models for scientific paper summarization with citation networks. In AAAI.

Klaus Zechner. 1996. Fast generation of abstracts from general domain text corpora by extracting relevant sentences. In Proceedings of the 16th conference on Computational linguistics-Volume 2, pages 986989.

Chrysoula Zerva, Minh-Quoc Nghiem, Nhung T. H. Nguyen, and S. Ananiadou. 2020. Cited text span identification for scientific summarisation using pretrained encoders. Scientometrics, pages $1-29$.

Jingqing Zhang, Yao Zhao, Mohammad Saleh, and Peter J. Liu. 2019. Pegasus: Pre-training with extracted gap-sentences for abstractive summarization. ArXiv, abs/1912.08777. 
Hao Zheng and Mirella Lapata. 2019. Sentence centrality revisited for unsupervised summarization. In ACL.

Ming Zhong, Pengfei Liu, Yiran Chen, Danqing Wang, Xipeng Qiu, and Xuanjing Huang. 2020. Extractive summarization as text matching. ACL. 